\title{
LA SECONDE SOPHISTIQUE ET LA PHILOSOPHIE
}

\author{
Jean-Luc Vix ${ }^{1}$ \\ jean-luc.vix@wanadoo.fr \\ "p I atwna tok twh rhtorwn patera kaivdidaskalon?" \\ Aelius Aristide, or. $2, \S 465$.
}

RESUMO Através do estudo de alguns discursos de Élio Aristides, este artigo visa esclarecer o lugar ocupado por Platão na formação do orador $e$, de maneira geral, determinar a importância que lhe foi dada no ensino da retórica na época imperial. Seria ele, realmente, o "pai e senhor dos oradores", como diz Aristides? Parece, com efeito, que os textos platônicos faziam parte da educação dos futuros oradores, elemento que, até o momento, não foi realmente objeto de estudos aprofundados.

Palavras-chave Elio Aristides; Platão; Retórica; Educação Retórica

RESUMÉ A travers l'étude de quelques discours d'Aelius Ariside, cet article veut discerner la place qu'a occupée Platon dans la formation du rhéteur et, plus généralement, déterminer l'importance qui lui a été accordée dans l'enseignement de la rhétorique à l'époque impériale. Était-il vraiment, comme Aristide le nomme, le "père et maître des orateurs»? Il semblerait, en effet, que les textes platoniciens faisaient partie intégrante de l'éducation des 
futurs rhéteurs, élément qui n'a, jusqu'à présent, pas réellement fait l'objet d'études approfondies.

Mots-clés Aelius Aristide; Platon; Rhétorique; Education Rhétorique

\section{Introduction}

Les relations d'Aelius Aristide, au $\mathrm{II}^{\mathrm{e}}$ siècle de notre ère, avec la philosophie ont souvent été analysées. Citons parmi tant d'autres les études, désormais datées mais qui restent indispensables de Boulanger (A.), Aelius Aristide et la sophistique dans la province d'Asie au IIe siècle de notre ère (Bibliothèque des Écoles françaises d'Athènes et de Rome, 126), Paris, 1923, et Wilamowitz (U. von), "Der Rhetor Aristeides", Sitzungsberichte Preussischen Akademie Wissenschaft, 1925, p. 332-353, ainsi que le chapitre consacré à Aristide et Platon dans Sohlberg (D.), Aelius Aristides und Diogenes von Babylon; zur Geschichte des rednerischen Ideals, Dissertation, Bâle, 1972, les réflexions de C A. Behr dans Aelius Aristides and the sacred Tales, Amsterdam, 1968 (p. 11, n.28), l'article de Moreschini (C.), "Elio Aristide tra retorica e Filosofia", dans ANRW, 34, 2, p. 1234-1247 ou l'étude plus générale consacrée à la "Rhétorique et philosophie au second siècle ap. J.-C.” par Michel (A.), dans ANRW, 34, 1, Berlin, 1992, p. 1-78. La recherche actuelle s'intéresse de plus en plus aux liens entre Aristide et Platon et la bibliographie proposée est très incomplète; ${ }^{2}$ mais la majorité des travaux sont généralement orientés dans deux directions: déterminer quels furent les conceptions philosophiques du rhéteur, -voire s'attacher à en démontrer l'absence -, et analyser sa polémique avec Platon. Les noms d'Aristide et de Platon évoquent, en effet, immanquablement les trois discours platoniciens du rhéteur, Sur la défense de la rhétorique, réplique au Gorgias, le Pour les Quatre, nouvelle réplique au Gorgias avec la défense de Miltiade, Thémistocle, Cimon et Périclès, Contre Capiton, controverse avec un philosophe contemporain qui faisait suite au discours Sur la défense sur la rhétorique. L'essentiel de ces discours concerne une réplique au Gorgias.

2 On pourrait encore citer LACY, Philiip de. Plato and the Intellectual Life of the second century. Appoaches to the second sophistic. Paper presented at the $105^{\text {th }}$ Annual Meeting of the American Philological Association, University Park, Pennsylvanie, 1974, p. 4-10; TRAPP. Plato's Phaedrus in second-Century Greek literature. Antonine Literature, 1990; FLINTERMAN, J. J. Largely fictions...: Aelius Aristides on Plato's Dialogues. Ancient Narative, 2001. 
Dans ce contexte, riche d'analyses pertinentes, notre objectif n'est pas de revenir ici sur les conceptions philosophiques d'Aristide ou sur sa polémique avec Platon. Nous voudrions, à travers l'étude de quelques discours, tenter de discerner la place qu'a occupée le philosophe dans la formation du rhéteur et, plus généralement déterminer l'importancel accordée à Platon dans l'enseignement de la rhétorique à l'époque impériale. Était-il vraiment comme Aristide le nomme le "père et maître des orateurs» (or. 2, §465: P I a t wn a tor twh rhtorwn patera kaivdidabkalon? Il semblerait, en effet, que les textes platoniciens faisaient partie intégrante de l'éducation des futurs rhéteurs, élément qui n'a, jusqu'à présent, pas réellement fait l'objet d'études approfondies.

Pour ce travail nous nous appuierons principalement sur cinq discours d'Aristide, les discours 30 à 34, sans renoncer à puiser, quand cela s'avérera nécessaire, à d'autres sources. Ces cinq discours, beaucoup moins connus que les discours platoniciens, n'ont jamais été étudiés sous cette optique. Pourtant ils peuvent nous apporter des informations intéressantes dans la mesure où ils sont tous, peu ou prou, en relation avec la paideia grecque et l'enseignement de la rhétorique tels que les vivait Aristide et les hommes de son temps.

\section{Présentation des discours:}

XXX: le Discours d'anniversaire à Apellas, un élève du rhéteur à Pergame, serait une œuvre de commande de la famille d'Apellas. Le jeune garçon (quatorze ans?) devait la même année présider des jeux en l'honneur d'Asclépios. Ce discours aurait été tenu en 147, ${ }^{3}$ alors qu'Aristide venait de passer ses deux premières années de soins dans l'Asclépieion de Pergame.

XXXI: Discours en l'honneur des funérailles d'Étéonée, un de ses jeunes élèves décédé à l'adolescence. Discours à la datation incertaine. ${ }^{4}$

XXXII: Oraison funèbre en l'honneur d'Alexandros: discours écrit à l'occasion du décès de son ancien maître, le grammaticos Alexandros de Cotiaeion.

XXXIII: A ceux qui lui reprochaient de ne pas déclamer: est une violente diatribe peut-être écrite en septembre $166,{ }^{5}$ sous forme d'une lettre à un ami, après une longue période d'inactivité oratoire liée à des problèmes de santé.

3 La date mentionnée, qui n'a aucun caractère de certitude, est celles retenue par BEHR, C. A. P. Aelius Aristides, The Complete Works. Translated into English, 2 v., Leyde, 1981-1986, T. 2, note 1 du discours XXX, p. 389.

4 Info lit.

5 BEHR, C. A. P. Aelius Aristides, The Complete Works. Translated into English. Leyde, 1981-1986, T. 2, note 1 du discours XXXIII, p. 396. 2 v. 
Aristide, non seulement répond à des accusations, mais s'attaque aussi au désintérêt de ses élèves.

XXXIV: Contre les profanateurs: violente diatribe contre ceux qui dénaturent l'éloquence.

\section{La formation d'Aristide: la place de Platon}

On ne sait pas grand chose de l'enseignement suivi par Aristide ni des maîtres qu'il eut. Nous avons une seule certitude à ce sujet: il a bénéficié dans son jeune âge des cours du grammaticos Alexandros de Cotiaeion, personnalité intellectuelle de premier plan qui deviendra le précepteur du jeune Marc Aurèle. Dans l'oraison funèbre rédigée à l'occasion du décès de son ancien maître (or. 32) Aristide, dans l'éloge qu'il fait du défunt, évoque les auteurs qui faisaient partie de l'enseignement du savant. Parmi ceux-ci on ne sera pas étonné de voir figurer les poètes ( $\$ 24$ Homère, Archiloque, Hésiode, Simonide, Stésichore, Pindare, Sappho, Alcée), "objets" traditionnels et privilégiés d'études de l'enseignant du cycle secondaire. Leur sont associés au paragraphe suivant, les auteurs en prose de façon générique sans qu'aucun nom soit cité, à l'exception notable de Platon dans une remarque qui est censée marquer la très grande proximité entretenue entre Alexandros et le philosophe: "Pour ma part, je riais de ceux qui mettent Platon en avant et ne philosophent qu'en se tournant vers celui-ci, alors que lui [Alexandros], à mon avis, davantage que tout autre, a été cher à Platon". ${ }^{6}$ Nous laisserons de côté la charge polémique contre les philosophes contemporains, ${ }^{7}$ généralement relevée dans les quelques commentaires sur ce passage, pour nous attacher à l'aspect relevant de l'enseignement. Il vaut la peine de s'arrêter sur cette remarque qui insiste sur la proximité du grammaticos avec le philosophe. L'enseignement de la rhétorique incluait-il, dès le niveau du grammaticos, une approche des philosophes? Notre connaissance de l'enseignement dans l'antiquité nous pousserait à répondre par la négative, mais la réflexion d'Aristide, ainsi que sa propre connaissance de Platon, semble aller à l'encontre de ces certitudes. Si la

6 WILAMOWITZ. Der Rhetor Aristeides, SPAW 1925, p. 350, affirme que Aristide a lu Platon (Gorgias et Phèdre) dans sa jeunesse auprès d'Alexandros. Dans l'article «Aristide» du Dictionnaire des philosophes antiques, p. 358 sqq., L. Pernot, "Platon contre Platon: le problème de la rhétorique dans les Discours platoniciens d'Aelius Aristide", dans Dixsaut (M.) (éd.), Le platonisme dévoilé (Tradition de la pensée classique), Paris, 1993, T. I, p. 315-338, souligne, quant à lui: "Aristide connaît bien Platon, certainement de première main: il le cite partout dans ses écrits, discute spécialement ses idées dans les discours II-IV et le voit même en rêve (L 57; LI 57-66)", (p. 361).

7 "Au cours de sa polémique contre Platon, Aristide a poussé plusieurs pointes contre les philosophes de son temps" (BOULANGER. Aelius Aristide..., p. 259). 
philosophie était incluse dans le champ d'étude des écoles de rhétorique, quel était le but de cet enseignement? S'agissait-il d'une réflexion approfondie sur les conceptions philosophiques, d'une sorte de culture générale qui se devait d'inclure l'étude des grands écrits philosophiques ou, de manière encore plus restrictive, d'une analyse stylistique, purement technique des textes des philosophes, au premier rang desquels Platon?

Avant de tenter de répondre à ces interrogations, essayons de discerner quelle place occupe Platon dans l'œuvre d'Aristide.

Bien que Wilamowitz ${ }^{8}$ et Behr ${ }^{9}$ supposent qu'Aristide ait suivi les cours d'un philosophe platonicien, Gaius, dont l'école était florissante à Pergame à cette époque, ${ }^{10}$ il semble à peu près exclu, au vu de l'œuvre du rhéteur, que ce dernier ait bénéficié d'un enseignement philosophique poussé car il ne cherche jamais à se situer sur le plan de la spéculation philosophique ${ }^{11}$ même s'il prétend, dans son discours Sur les Quatre (or. 3, §690) qu'il a étudié avec les meilleurs philosophes de son temps. ${ }^{12}$ Dans ses discours platoniciens il situe la controverse essentiellement sur le terrain de la rhétorique. Il semble assuré, d'autre part, au vu des citations, que seul Platon a été étudié par Aristide. ${ }^{13}$ Wilamowitz tout en reconnaissant au rhéteur une bonne connaissance du Gorgias et du Phèdre pense que sa familiarité avec l'œuvre du philosophe n'était pas très grande, ce qui, on le verra est très discutable. Moreschini, quant à lui, souligne que rien ne permet d'affirmer que Aristide ait eu une connaissance étendue de la philosophie platonicienne. ${ }^{14}$ Cependant, tout indique une grande proximité du rhéteur avec l'œuvre de Platon, si ce n'est avec sa philosophie. En effet, ce dernier est un des auteurs les plus volontiers cités dans les discours aristidiens. Si l'on s'en tient au décompte opéré par Behr ${ }^{15}$ on constate une très grande diversité de passages platoniciens apparaissant dans les textes d'Aristide: Apologie(20), Phédon(5), Cratyle (4), Théétète (4), Politique (2), Parménide(1), Banquet (19), Phèdre (25), Alcibiade (5), Théagès (1), Lachès (2), Lysis(1), Euthydème(2), Protagoras (17), Gorgias (180), Ménéxène (14, République (53), République (53), Lettre VII (10). Encore faut-il considérer

8 op. cit. p. 350.

9 op. cit. p. 12.

10 Opinion réfutée par C. Moreschini, “Elio Aristide tra retorica e Filosofia”, dans ANRW, 34, 2, p. 1234-1247., p. 1242.

11 L. Pernot, “Platon contre Platon ... 1993, p. 327: “Aristide n’est pas philosophe, cela est trop évident, et son platonismene ne résiste pas à l'examen.".

12 oimai devkagwVsuggenesgai twh ep emautou'filosofhsantwn toi's ajistois kaivteleuwtavois. Encore faut-il souvent entendre le terme de philosophes chez Aristide comme homme de bien.

13 cf. BEHR, op. cit., p. 11

14 Loc. cit., p. 1241.

15 Op. cit., p. 11, n. 28. 
que cette liste est restrictive car ne sont répertoriés que les citations et ignore toutes les allusions plus ou moins cachées, fort nombreuses. Et pourtant, quelle variété! Ce ne sont pas un ou deux dialogues que notre rhéteur avait étudié, mais manifestement une grande partie de l'œuvre du philosophe. Nous retrouvons cette richesse dans le corpus des cinq discours 30-34, puisque, d'après un décompte que nous avons effectué, les allusions, ciations références à Platon se distinguent de la manière suivante: Apologie (6), Charmide (1), Euthydème (1), Gorgias(12), Lettre VII (1), Lois (6), Lysis (1), Ménéxène (1), Phédon (3), Phèdre (10), Philèbe (1), Politique (1), Protagoras (2), République (10), Théétète (3), Timée (1).

Platon, auquel il ne ménage pas son admiration, fait donc partie intégrante des valeurs littéraires revendiquées par Aristide. Il n'est à ce titre, sans doute pas anodin qu'il le voie en rêve ainsi qu'il le relate dans les Discours sacrés, IV, $\S 58$ et V, §57-63. Dans le DiscoursV, $\S 58$ un jeune homme, faisant la louange du rhéteur le compare à Platon: $0 u \forall 0 s$, ef $h$, es t i VP I a tw $n$ ka i VQ oukudidh $\varsigma$ ka iVP I a twn kaiVoJdeiha, ka iVool I ou su z eugnu rs t ina, "Cet homme-là c'est Platon et Thucydide, Platon et Un tel, et il en énuméra ainsi beaucoup, attachant chaque fois un autre nom à Platon". Le philosophe apparaît dans ce songe comme une sorte de quintessence de la prose grecque et comme la figure essentielle du patrimoine culturel hellène.

\section{Platon, figure du patrimoine culturel grec}

\section{a. La vertu platonicienne}

Aristide associe volontiers Platon à Pindare dans nos discours. Ainsi au discours 34, §5-6, le philosophe est mentionné aux côtés du poète en tant que caution morale, à l'usage de la démonstration du sophiste qui, dans son attaque contre les mauvais orateurs, veut prouver qu'on ne peut être méchant ou médiocre de son plein gré. Les deux auteurs y sont qualifiés de sages entre les sages (PI atwn kaiVPindaros pollach' mell kaiVa[lh/s of oi ) et leur convocation comme témoins rappelle le rôle joué par Homère dans la littérature impériale, mais aussi dès l'époque classique, celui d'une valeur morale inattaquable. ${ }^{16}$ Platon est, lui aussi, garant d'une morale. A titre d'exemple il faudrait citer l'ensemble du $§ 20$ du discours 30 dans lequel le rhéteur cite

16 Simonide apparaît comme l'un des auteurs anciens auquel les Grecs se réfèrent le plus volontiers avec Homère et Hésiode "pour leur emprunter enseignements moraux ou réflexions philosophiques" (Revue des Études Grecques, 88, p. 20). 
un long passage des Lois, "manière de placer sous le patronage de Platon l'ensemble des qualités morales du jeune garçon dont il fait l'éloge ${ }^{17 "}$ ". Cette citation comporte un grand intérêt pour notre propos. Non seulement Aristide use très rarement de longues extraits d'auteurs dans son œuvre, mais il insiste ici particulièrement sur le fait qu'il cite de mémoire sans document à l'appui: kaiVgav pws efucon autwh a pomnhmoneusas, ouf vafelwb oudeb oufe prosqei ajouter". On connaît l'importance de la mémoire dans l'éducation antique; les élèves apprennent par cœur des passages importants, en particulier de poètes. ${ }^{18}$ Rien ne permet donc de mettre en doute la parole du sophiste. Cet aspect semble confirmer que Platon faisait effectivement partie des auteurs étudiés dans les écoles de rhétorique. Toujours dans le cadre de l'éloge de son jeune élève Apellas, Aristide, au $\S 22$, va à nouveau faire appel au philosophe, sans le citer textuellement mais en se référant cependant expressément à lui pour parfaire le portait de son élève: "Voilà ce qu'est une nature pure, - composée de cire non mélangée selon l'expression de Platon ${ }^{19}-$, comportant une composition intermédiaire entre l'humide, le mou, le sec et le pierreux."

Mais l'influence de Platon ne se limite pas aux seules citations dans ce domaine très spécifique de la philosophie qu'est la morale. De même que dans le Discours pour les Quatre Aristide trace le portrait de l'orateur parfait, tel qu'il s'est réalisé dans les quatre personnages (Miltiade, Thémistocle, Cimon, Périclès) en faisant la part belle aux qualités morales, de même nos cinq discours dessinent-ils l'orateur parfait qui vise le beltiston en toutes circonstances. Dans le discours 34, véritable manifeste du bon orateur, les termes d'excellence (agathon, beltiôn, beltiston) sont innombrables. L'orateur parfait est un homme de bien qui possède les vertus de la tétrade platonicienne: courage, justice, prudence et tempérance. Ainsi voit-on dans les trois éloges individuels des discours 30 à 32 Alexandros loué pour sa justice (or. 32, §18 et 28), de même que le jeune Apellas, destiné à assurer le rôle d'agonothète des jeux en l'honneur d'Asclépios (or. 30, § 21). La sophrôsunê est constamment mise en avant, chez le jeune Étéonée (or. 31, §1,5) associée à la justice (§5),

17 PERNOT, L. La rhétorique de l'éloge dans le monde gréco-romain, T. I: Histoire et technique; T. II: Les Valeurs. Paris, 1993, T. II, p. 599.

18 Il faut avoir à l'esprit à ce sujet le début du Phèdre lorsque Socrate suppose que son interlocuteur a appris par cœur le discours de Lysias dont il veut l'entretenir (Phèdre, 228 b).

19 Aristide a vraisemblablement à l'esprit le passage du Tééthète, 194c-194e. Mais il ne cite pas, cette fois, textuellement Platon. Le jeu de motej a kh r a vo u ge to u 'kh r o u'n'y figure pas. Platon fait d'abord le portrait de l'âme parfaite et pure des sages, imprégnée de cire profonde abondante, lisse et pétrie comme il faut (194c-194d). Puis, en contrepoint il évoque les âmes composés de cire impure et très sèche, molle ou dure. 
chez Alexandros (or. $32, \S 15$ et 18) tandis que son absence est déplorée chez ses mauvais élèves (or. $33, \S 28$ ). Il n'est pas jusqu'à l'andreia qui ne soit chantée chez le jeune Étéonée (or. $31, \S 1$ ), aussi bien que chez le grand-père d'Apellas (or. $30, \S 12$ ). Le bon orateur est aussi celui qui se détourne des occupations triviales, comme Étéonée ou Apellas qui malgré leur jeune âge, refusent de s'adonner aux jeux des enfants. et, si les élèves d'Aristide sont vilipendés dans le discours 33, c'est précisément parce que, au lieu de consacrer leur temps à la rhétorique, ils cultivent les futilités, en passant par exemple leurs journées aux bains. L'orateur tel que le conçoit Aristide ne se complaît pas à satisfaire le public, comme le font les mauvais orateurs décriés dans le discours 34, mais a, au contraire, une mission d'éducation.

La vertu platonicienne se retrouve parfois également au détour d'un mot. Dans le discours $30, \S 18$ on trouve le terme de qu mo ei d e $\$$, terme platonicien ${ }^{20}$ que l'on repère dans le Timée, 18 a: cette "ardeur" doit se conjuguer avec la "modération" (f ilog of on) dans l'âme des gardiens de l'État selon Socrate. Le parallèle est d'autant plus probant que, suivant les conseils du philosophe, Aristide souligne que l'ardeur du jeune garçon dont il fait l'éloge, caractéristique de la nature masculine, est tempérée par une certaine forme de douceur.

Certes, on peut rétorquer que la mention de ces vertus faisait partie, à l'époque impériale des topoi mis en œuvre dans toute œuvre encomiastique. Il n'en reste pas moins qu'ils sont directement influencés par la pensée du philosophe. En outre, Aristide n'hésite pas à récupérer, pour les besons de ses démonstrations, certains concepts platoniciens.

\section{b. La récupération des concepts platoniciens}

Aristide, par exemple, emprunte à Platon ses théories sur l'immortalité de l'âme quand cela s'avère nécessaire, ce qui se justifie plus qu'ailleurs, on le comprend, lors de l'éloge d'un mort.

Dans le $\S 16$ du discours 31 le rhéteur évoque l'immortalité promise au jeune Étéonée. Nous avons déjà signalé qu'Aristide utilise ce thème comme un topos rhétorique de consolation sans y adhérer lui-même. Pourtant la référence à Platon s'impose d'emblée à lui: thW a poikian ouk aheu qei pepo i meno n, "il n'a pas entrepris le voyage sans un privilège divin". La theia moira est une expression typiquement platonicienne (par ex. Phédon, 58e: mhd veijs Aidou ijota aheu qei $s$ moira $s$ ifuai, "ne pas aller vers la 
demeure d'Hadès sans un concours divin". L'image du voyage qui apparaît dans ce passage est annoncée dès le paragraphe précédent: o u d e Vel eei q qa i

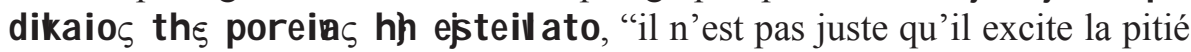
pour le voyage qu'il a entrepris", métaphore platonicienne cf. Platon, Apologie, $40 \mathrm{e}$, qui certes était aussi largement utilisée à l'époque impériale dans les inscriptions funéraires. D'autres parallèles pourraient être établis entre la fin du jeune garçon et celle de Socrate telle qu'elle est décrite par Phédon: dans les deux cas le défunt apparaît comme un bienheureux que la félicité attend. C'est donc, à travers des expressions et des images, tout un fond platonicien qui est discrètement mis en place par l'orateur.

Dans l'oraison funèbre d'Alexandros, (or. 32, §34) Platon apparaît en compagnie de Pindare dans une allusion concernant l'immortalité de 1'âme: "Si les paroles de Pindare, de Platon, et de l'ensemble de l'atelier d'Alexandros sont vraies, et s'il existe quelques entretiens entre les occupants de l'Hadès [...]". Platon effectivement évoque les conversations des défunts dans l'Hadès. ${ }^{21}$ La préoccupation d'Aristide n'est pas un débat de fond sur la théorie platonicienne, à laquelle il était vraisemblablement hermétique; ce qui lui importe c'est d'apporter le témoignage du philosophe au même titre que celui du poète: la caution de la tradition littéraire. L'un et l'autre appartiennent au panthéon littéraire de tout pepaideumenos, tout grec cultivé.

Mais le philosophe est aussi mis à contribution dans des domaines plus profanes. Toujours dans le même discours 32, §26 il apporte son soutien à l'orateur qui veut prouver que les écrits savants de son ancien maître n'étaient rien comparés à ses cours: w\$t vemo i ge to Vt ou P I a twno s a uf. ou paristasqai, paidiall eihai dokein taven tois grammasi proł ta $\$$

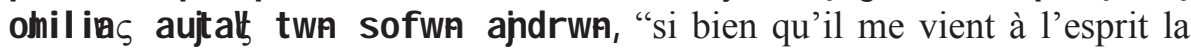
remarque de Platon lui-même que ce qu'il y a dans les écrits semble être un jeu $^{22}$ comparé aux entretiens eux-mêmes des sages" (Phèdre 277e). On n'est plus du tout dans le cadre d'une citation mais bien d'une sorte de synthèse d'un paragraphe du discours platonicien. Davantage, Aristide, adapte la pensée de Platon pour l'intégrer dans son propos, puisque nulle part le philosophe n'évoque les entretiens des sages, et qu'il parle de ce qui est enseigné et des propos destinés à l'instruction. Sans doute le rhéteur n'avait-il plus à l'esprit le texte exact du Phèdre, ce qui l'a obligé à faire cette adaptation, mais seule une

21 Apologie, 4la-c.: Le philosophe y évoque la possibilité d'y vivre en compagnie d'Orphée, de Musée, d'Hésiode, d'Homère et de bien d'autres, avant d'ajouter que ce serait le lieu d'une merveilleuse conversation, qaumasth Va hi ei h diatribhv(41 a).

22 Aristide profitant de cette citation du Phèdre (277e), joue, à nouveau, avec les mots puisqu'ici pa idia vfait écho à paidei g $(26,22)$. 
bonne connaissance de l'œuvre a rendu cela possible. Ce qui nous l'indique c'est, d'abord, l'emploi de ei $₫$ w $\mid$ a a dans la phrase précédente, mais sans aucune mention de Platon: K a iVmh toutovge oug vuma's I anganein eiko $\$$ oufe touł u\{teron wh kalaVmell kaiVa\}gray a skatevipe kaiVbeltiona twh omof uvwn pantwn, ofnw $s$ deVmikraVafta eidwla th's ekeinou paidei a $s$ te kaiVgnwmh $\varsigma_{1}$ "D'ailleurs cela ne vous échappe sûrement pas, ni à vous ni à la postérité: les écrits qu'il laissa sont bons sans doute, et meilleurs que ceux de tous ses congénères, mais ne sont cependant que de pâles images ${ }^{23}$ de son enseignement et de sa pensée." Or, l'opposition: parole/écriture est mise en relation avec l'opposition, centrale dans la philosophie de Platon, modèle/ image ce qui fait dire au personnage de Phèdre (276 a) que le discours écrit n'est qu'un simulacre ( $(\mathrm{\downarrow}|\mathrm{w}| \mathrm{O} n)$ du discours parlé. Aristide avant de feindre la spontanéité ("il me vient à l'esprit la remarque de Platon") et de citer le philosophe avait, en réalité, déjà instillé l'essentiel de sa pensée, en la reprenant à son compte.

On retrouve, ensuite, une trace du même passage du Phèdre dans un autre discours, 30, §19. Nous avons vu que le jeune Apellas est plusieurs fois placé sous l'ombre tutélaire du grand philosophe, gage de sa sagesse présente et à venir. Ce sophos en devenir sait allier le sérieux et le jeu nous dit le rhéteur, ajoutant: a I I a Vpah, kah hXcalepol, ouk ekt in eprth'aheu pracqhhai, "rien, même la difficulté, ne peut être fait sans un esprit de fête". Cette remarque n'est pas annoncée comme une citation, le nom de Platon n'apparaît pas. Pourtant on trouve dans le Phèdre 276 b: pa i d i a 's t e ka i Vepr th's ca vi in $d r w$ a h, oke ka iVpoi o i " "S'il lui arrivait de le faire, ne serait-ce pas par jeu et à l'occasion de la fête?". Nous sommes donc directement dans la suite de la réflexion générale sur le discours écrit, simulacre du discours oral. "Dans la philosophie platonicienne l'opposition écrit/oral en appelle une autre: sérieux/ jeu". ${ }^{24}$ Mais, bien que la réflexion de Socrate s'inscrive dans une image, destinée à illustrer la supériorité de l'oral sur l'écrit, ce souci n'apparaît à aucun moment dans le discours d'anniversaire en l'honneur d'Apellas. Aristide

23 L'emploi de ei $\downarrow$ wla renvoie à Platon, Phèdre, 276 a, passage dans lequel le personnage de Phèdre est amené par Socrate à dire que le discours écrit n'est qu'un simulacre ( $(e i \downarrow w \mid$ on $)$ du discours parlé qualifié de discours vivant et animé. Brisson (L.), Phèdre, traduction introduction et notes, (GF Flammarion, 488), Paris, 1989, p. 232, n. 450: “L'opposition: parole/écriture est mise en relation avec l'opposition centrale dans la philosophie de Platon: modèle/ image. Cette opposition en appelle une autre: sérieux/ jeu" (Phèdre 276 b) qui est, elle, utilisée par le rhéteur dans le discours d'anniversaire en l'honneur d'Apellas (§19) mais sans que le lien avec la problématique écrit/oral y apparaisse. L'ensemble du paragraphe est donc imprégné par la pensée platonicienne, si bien que l'apparente spontanéité du sophiste dans la suite du paragraphe "il me vient à l'esprit la remarque de Platon lui-même" ne peut être considérée, bien entendu, que comme un artifice rhétorique.

24 BRISSON, L. Phèdre, traduction introduction et notes, (GF Flammarion, 488), Paris, 1989, p. 232, n. 450. 
se contente de reprendre l'esprit de la remarque du Phèdre en la détournant totalement de son sens originel pour faire l'éloge de son jeune élève qui sait allier sérieux et amusement. Peut-être est-ce Aristide qui s'amuse lui-même ici! Étrange dialogue intertextuel entre ses discours, à travers ces références platoniciennes.

Mais, avec ce dernier exemple nous sommes déjà dans une référence textuelle détachée de son contexte. Platon n'est plus le philosophe dont on peut reprendre tel ou tel concept pour sa propre démonstration, mais bien le prosateur dont on copie tel ou tel tour, jugé élégant, telle ou telle image considérée comme frappante.

\section{Platon l'écrivain}

Nous nous proposons donc dans cette dernière partie de mettre en valeur le travail d'Aristide écrivain, inspiré par Platon le prosateur. Il arrive souvent que le rhéteur puise dans le fond lexical du philosophe sans jamais préciser dans ce cas, ses sources. Aussi ces passages sont-ils plus difficiles à repérer.

Il est intéressant, d'abord, de noter que notre sophiste n'hésite pas à utiliser la forme du dialogue pour persuader ses auditeurs, mimant partiellement la dialectique platonicienne. ${ }^{25}$ Le discours 34 est à cet égard particulièrement riche. En effet, l'essentiel de la fin du discours se déroule sur ce mode, alternant questions-réponses, démonstrations-réfutations, §58- 59: "Mais, puisque nous faisons grand cas même de la gloire, pour le souvenir futur, à quel rang pourrait-on placer ces hommes dont la réputation meurt chaque jour et sur-le-champ? (59) Oui!, ${ }^{26}$ par Zeus, mais (nh VD i va I a V même Héraklès a dansé chez les Lydiens. Certes, mais ( 0 i deVg va uf. o i V les mêmes auteurs racontent aussi ce mythe au sujet d'Héraklès: il tua sa femme et ses fils, alors qu'il était dans un état qu'il n'est pas permis de nommer. ${ }^{27}$ Quelle personne sensée pourrait être persuadée par cela? Toi en tout cas, si vraiment tu y crois, réponds-moi, au nom d'Héraklès lui-même, si toi-même, pour cette raison, tu détruirais volontiers ce qui t'appartient? Tu répondras que non." Le principe du dialogue, on le constate, n'est pas poussé au bout de sa forme puisque c'est Aristide qui répond à la place de ses interlocuteurs fictifs à la fin, comme cela

25 Cela correspond aussi au procédé très caractéristique de la diatribe, de l'ajpqupof or avqui consiste à soulever l'objection d'un objecteur fictif ou à prévenir celle de l'interlocuteur pour mieux amener la solution recherchée.

26 Aristide crée un dialogue fictif. Ici, il donne la parole aux sophistes qu'il critique.

27 Selon Behr,The complete works, T.II, p. 399, n.45, il est impossible de parler de sa folie, parce que c'est un dieu. Il propose comme parallèle L'hymne à Poséidon, (or. 46), §32-41. 
se produit également chez Platon (cf. la fin du Gorgias). La mimesis de son illustre ancêtre est clairement perceptible.

Parfois ce sont quelques mots qui marquent discrètement une allégeance et une dette à l'égard de l'écrivain Platon. C'est le cas dans le discours 33, $\$ 31$ avec l'emploi du verbe kulindwmeqa: kaiVmh VthW u£ion a monian hjmosmenoi nukta ek nukto $\$$ kaivhmeran ej hmeras kulindwmeqa, "et afin d'éviter que après avoir réalisé l'harmonie des porcs, nous ne nous y vautrions nuit après nuit et jour après jour". Nous retrouvons des parallèles dans le Phèdre 257 a (l'âme de celui qui n'est pas vraiment aimé, condamnée à rouler neuf mille ans autour et sous la terre) et avec un passage du Politique 309 a, (mention des caractères qu'on ne peut éduquer, qui se "vautrent dans l'ignorance et l'abjection"). L'expression péjorative (t ou $\downarrow$ d $d$ v e h a ma qi a $[\ldots] / \mathrm{k} u \mathrm{I}$ in d o u men o u $\varsigma$ ), qu'on trouve dans ce dernier extrait, figure, du reste, également dans le Phédon, 82 e: é pash / a maqia/kulindoumenhn (sc. y u c h n ), "elle était vautrée dans une ignorance absolue". Il n'est peut-être pas non plus dû au hasard que l'on retrouve ce verbe dans la bouche de Socrate (Phèdre $275 \mathrm{~d}$ ) lorsqu'il décrit de façon imagée le discours écrit: "quand une fois pour toute, il a été écrit, chaque discours va rouler de droite et de gauche et passe indifféremment auprès de ceux qui s'y connaissent, comme auprès de ceux dont ce n'est point l'affaire". Le verbe a ici les deux acceptions de "rouler" et "se vautrer" qui peuvent aussi lui être attribuées dans notre passage. Nous avons déjà relevé plus haut combien cette problématique du Phèdre était connu du sophiste.

Les textes platoniciens surgissent parfois également au détour d'une image

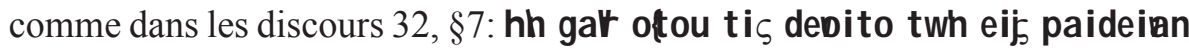
par vekeinou I a mbanein, w\$per ek phgwh a juomenon, "car il était possible d'obtenir auprès de lui tout ce dont on avait besoin en vue de la culture, comme on puise de l'eau aux sources". La comparaison avec la source n'est pas une originalité de notre sophiste mais appartient à la tradition littéraire grecque, et serait d'inspiration platonicienne. Le philosophe emploie très couramment la métaphore de la source au sens de commencement, principe de quelque chose, par ex. dans le Phèdre $245 \mathrm{c}$, l'être de qui procède tout est qualifié de "source et principe de mouvement", dans le Timée 70 b c'est le cœur qui est "source du sang" "28. Cette métaphore a été reprise dans tous les domaines, on la retrouve par ex. chez Longin, Du Sublime, VIII, 1, qui évoque certains styles, sources du sublime. Dans les deux exemples platoniciens ci-dessus, la source est 
principe mais aussi synonyme de vie, de même que l'ancien maître d'Aristide était source de vie intellectuelle, voire spirituelle pour ses contemporains. Mais le parallèle pourrait sembler fragile sur cette seule idée car le rhéteur, dans notre passage, n'emploie pas l'image de la source dans le sens que lui donne habituellement Platon. Pourtant, c'est chez ce dernier que l'on trouve une métaphore approchante, dans le Phèdre, 253 a: ka jn ek Dio $\$$ a j u t w s in, "s'ils puisent à la source de Zeus". Il ne s'agit pas d'une coïncidence: Aristide qui possède son Platon opère une substitution pour adapter la référence à son discours. Le nom du grand dieu est remplacé par cette image si courante chez Platon, la ph gh v Ce faisant il mêle la métaphore platonicienne au verbe a j u w qui apparaît dans le passage du Phèdre. Les amants puisent à la source de Zeus pour reverser ses bienfaits sur leur bien-aimé, comme les Grecs puisaient à la source du savoir d'Alexandros pour s'en enrichir.

Pour terminer il faut signaler que l'imitation de l'œuvre de Platon peut aller jusqu'à une sorte d'identification avec Socrate. Dans le discours 33 Aristide est obligé de se défendre face à des adversaires qui lui reprochent de ne pas déclamer, autrement dit de ne pas assumer son travail d'orateur et d'enseignant. Pour répondre à cette accusation le sophiste va très subtilement se glisser dans la peau de Socrate. Comme ce dernier il est accusé injustement, comme ce dernier il veut se défendre. Al'inverse du philosophe il ne risque pas d'être condamné à mort. Le premier indice se trouve au $\S 3$ dans l'expression skiamacein meb ouh pws oib volfi dei, probs ous gat eijhsetai tav l egomena ouj hkous in, “je sais bien qu'il me faut combattre comme contre des ombres ${ }^{29}$ car ils ne sont pas là, ceux que ces propos viseront". Passage qu'il faut confronter à Apologie, $18 \mathrm{~d}$ : ajpagk af ecnw's w\$per skia maceih a pologoumenon te kaiVejegcein mhdenobapokrinomenou, "je suis tout bonnement forcé, en me défendant, de me battre, en quelque sorte, contre des ombres et de réfuter sans que personne me réponde!". Le parallèle semble suffisamment évident pour qu'il ne soit pas nécessaire d'y insister. Mais Aristide n'en reste pas là; dans le même paragraphe il commence sa défense en utilisant des termes juridiques comme s'il était devant l'Assemblée des

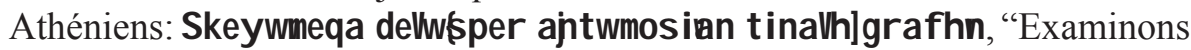

29 Même emploi du verbe s kia ma cein dans le discours Sur la digression, or. 28, §1. Il s'agit d'une référence platonicienne, Apologie, $18 \mathrm{~d}$ : Socrate déplore ne pas connaître ses accusateurs et être obligé de combattre comme contre des ombres (w \$ per skia mac cih). La présence de ce verbe n'est pas fortuit car il est évident que Aristide a l'Apologie de Socrate à l'esprit ainsi que le terme d'a hit w mos i a (Apol., 19 b) qu'il utilise à la fin du paragraphe le montre. Tout le discours est placé sous le signe d'une défense, avec plusieurs emplois de termes juridiques et le mot d'apologi в lui-même au dernier paragraphe. En établissant un parallèle entre sa situation et celle du philosophe condamné injustement à mort il cherche à souligner la gravité des accusations portées contre lui, mais aussi leur inanité. 
donc (l'accusation) comme s'il s'agissait d'une sorte de déclaration sous serment ${ }^{30}$ ou d'une d'action publique". Le terme d'antwmosi appartient spécifiquement au droit attique et apparait dans l'Apologie, $19 \mathrm{~b}$, peu après le passage signalé ci-dessus et à proximité du terme de graf $\mathrm{h} v \mathrm{w}$ \$per ouh kathgorwn thw ajtwmosian dei'ajhagwhai autwh, "faisons comme si, en leur qualité d'accusateurs, ils avaient accompagné la plainte du serment légal" il faut donc donner lecture de la plainte. ${ }^{31}$ Aristide, comme Socrate, use donc de l'artifice du procès pour assurer sa défense. Ce travail d'identification sera parachevé au dernier paragraphe (\$34) du discours avec l'apparition du terme totalement dénué d'ambiguité de a p ol 0 gi a pour qualifier son discours. ${ }^{32}$

\section{Conclusion}

Ce corpus des cinq discours est tout à fait spécifique dans l'œuvre du rhéteur. En effet, si Platon, comme cela a souvent été relevé, est constamment présent dans l'ensemble de ses discours, les cinq discours pris en compte, présentent à cet égard un intérêt particulier dans la mesure où ils offrent sous des angles multiples une vision de l'enseignement de la grammaire et de la rhétorique à l'époque d'Aristide et telle qu'elle était vécue par lui. Le platonisme dont on détecte une présence si constante et importante y est un indice de la présence du philosophe dans l'enseignement de la rhétorique et dans les milieux éducatifs, à côté des poètes, historiens et orateurs qui sont cités habituellement comme faisant partie de cet enseignement. Ces traces si importantes du platonisme dans ces cinq discours, ne peuvent, par conséquent, être mises en parallèle avec celles que l'on trouverait dans les discours platoniciens dans lesquels Aristide engage une polémique avec le philosophe.

La connaissance qu'a Aristide de Platon est bien plus complète qu'on n'a pu le dire parfois. Ces quelques exemples, qui sont parmi les plus significatifs, soulignent à quel point l'œuvre du philosophe est familière à notre sophiste. Cet art consommé de la référence allusive est le fait d'un homme qui a longtemps fréquenté les discours philosophiques et qui s'en est imprégné, de

30 Dans un procès, à Athènes, le demandeur et le défendeur après avoir déposé par écrit étaient tenus de prêter serment dès le début de l'anakrisis. Il s'agit de l' a ht w mos i i , terme qu'utilise Aristide ici. Mais le mot a pris, par extension, le sens d'accusation, comme on peut le voir chez Isée, Dikaiogenes, 2 et 4 . Voir: MEIER, M. H. E.; SCHÖMANN, G. F. Das Attische Recht und Rechtsverfahren mit Benutzung des Attischen Processes. Ed. Lipsius J. H. Leipzig, 1905-1915. p. 830.

31 Trad. L. Robin et M. J. Moreau.

32 On retrouve un parallèle avec l'Apologie (41 a) dans le discours $32, \S 25$, au sujet de l'immotalité et des entretiens que l'on peut avoir dans l'Hadès. 
leur pensée, comme de leur style. Aristide a lu Platon comme un prosateur, on devrait dire comme un orateur? Cette lecture approfondie a dû, au moins en partie, s'effectuer dès l'école du rhéteur, ou même dès le grammaticos. Si l'enseignement des grands poètes, puis des orateurs et historiens de la tradition est clairement attestée il semble bien que la philosophie n'en était pas absente.

Mais c'est, au vu de ce qu'on a pu constater dans ces quelques exemples, une philosophie à l'usage des rhéteurs. En effet, la pensée platonicienne, largement divulguée, fait partie intégrante de la culture grecque sous forme d'une vulgate reprise par les orateurs de l'époque impériale, voire dans les inscriptions funéraires. Aristide également en use volontiers mais il va au-delà. En tant que rhéteur on a le sentiment que, plus que les concepts philosophiques, ce qui l'attache à Platon c'est le goût de l'écriture et le plaisir de ce style qu'il avait appris à connaître à l'école du rhéteur.

Démosthène, en particulier dans les déclamations, reste le modèle incontestable, d'Aristide (cf. Discours sacré V, 62-63: il voit en rêve un temple dédié à Platon avec une statue de Platon.'Quelqu'un disant que Platon du moins eût dû avoir trois temples: «Et Démosthène, repris-je par surenchère, pourquoi pas quatre-vingts, et autant, je pense Homère!»”). Platon, pourtant semble largement occuper la première place des prosateurs dans les discours étudiés. Aristide avait de toute évidence deux maîtres de rhétorique, depuis l'enfance, comme il le proclame lui-même dans le discours Contre Capiton. ${ }^{33}$ Cette réflexion, loin d'apparaître comme un propos de rhétorique doit, au contraire, être comprise au sens propre ainsi que l'attestent nos discours.

33 Contre Capiton, §6: outw $\varsigma$ emoi koinw's mell eipeih twh palaiwh ajdrwh, di vakribeia $\varsigma$ devtoutwn upet

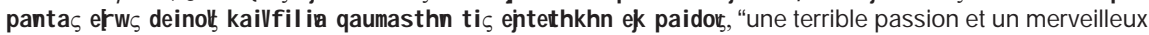
amour sont à ce point profondément entrés en moi depuis l'enfance pour les anciens en général, pour ainsi dire, et particulièrement pour ceux-ci [Platon et Démosthène] au-delà de tous". L'identification de Platon et Démosthène derrière le démonstratif toutwn est unanimement aceeptée depuis Reiske et ne semble pas devoir être remise en cause, voir: BEHR. The complete Works, T. I, p. 479, n. 11; SOHLBERG, D. Aelius Aristides und Diogenes von Babylon. Bâle, 1972. p. 183, n. 4. 